\title{
Muscle Atrophy in a Case of Acute Disseminated Encephalomyelitis
}

\author{
Shiro Matsubara, MD, Ph D, Yasunori Tabuchi, MD, Taneyoshi Nozawa, MD \\ and Hitoshi Tanabe, MD
}

\begin{abstract}
A case of acute disseminated encephalomyelitis (ADEM) accompanied by muscle atrophy is reported. A man of 30 was admitted with acute onset of clouded consciousness, fever, paraplegia and sensory loss, following an upper respiratory tract infection. A week later, marked muscle atrophy was noticed in both legs. A biopsy of the quadriceps femoris muscle showed groups of small angular fibres indicating neuropathic change, and also fibres with round contour and varying diameter suggestive of myopathic change. Some fibres contained vacuoles, basophilic material and or eosinophilic mass. Histochemical examinations suggested that the basophilic material contained mitochondria, lipid and glycogen, while the eosinophilic mass was regarded as hyaline degeneration. Cellular infitration was not observed in the interstitium. Electron micrographs showed disarray and loss of myofibrils, abundant mitochondria and glycogen granules, myelin figures and honeycomb structures. The cause of the muscle changes is uncertain. However, as conceivable causal mechanisms, the possibility of polymyositis is discussed as well as the effect of immobilization and probable involvement of the nerve roots and peripheral nerves.
\end{abstract}

Key Words: Histochemistry, Neuropathy, Myopathy, Polymyositis, Disuse atrophy, Regeneration of the muscle.

Muscle atrophy is known to be one of the uncommon signs of $\mathrm{ADEM}^{1,2,3)}$, but the histological changes in the muscle accompanying this disorder have not been fully investigated. We report a case of ADEM with muscle atrophy and describe the histological, histochemical and ultrastructural findings of a muscle biopsy.

\section{REPORT OF THE CASE}

History: A man of 30 , a civil servant, was admitted with clouded consciousness on 29 th November, 1974. He had been well until two weeks before when he first noticed a sore throat and a low grade fever. In the following two weeks, he had developed severe back pain, headache and stiffness of the shoulders. Despite of these symptoms, he continued his work. On the day before admission, all his symptoms got worse rapidly and his temperature rose to $38^{\circ} \mathrm{C}$. He noticed some confusion in his own conversation. Difficulties in walking and micturition began in the evening. Next morning he had generalized convulsions of a few minutes' duration and became unconscious. His past and family history were noncontributory.

Examination: On admission, he was stuporous and had a temperature of $38.7^{\circ} \mathrm{C}$. Both legs were paralysed. Muscle tone was normal in the upper limbs and reduced in the lower limbs; so were the tendon reflexes. The abdominal reflexes were absent and the plantar responses were equivocal. There was a loss of sensation to all moda-

From Department of Neurology, Toranomon Hospital, Tokyo.

Received for publication September 3, 1979.

Reprint request to: Shiro Matsubara, Department of Neurology,

Toranomon Hospital, 2-2, 2 chomeToranomon, Minatoku, Tokyo, 107, Japan. 
lities of stimuli below the level of Th. 1 . Moderate stiffness of the neck was noticed. There were mild rales over the lungs; no other abnormality was noted on general physical examination. No muscle atrophy was noticed at the time of admission.

Investigations: The WBC count was $10700 / \mathrm{cu}$. mm, the RBC count was normal; the ESR was $47 \mathrm{~mm}$ in 1 hour. The serum protein, serum electrolytes and BUN were all normal. The activities of serum LDH, GOT and GPT were normal; the serum creatine phosphokinase (CPK) activity was 18 units (Modified Rosalki method. Normal; 18-86). The serologic tests for syphilis were negative. The stool and urine were normal. The cerebrospinal fluid was yellowish and contained 291 cells/cu. $\mathrm{mm} ; 142$ of them were polymorphs and 149 mononuclear cells. It contained $221 \mathrm{mg} / 100 \mathrm{ml}$ of protein and $54 \mathrm{mg} / 100 \mathrm{ml}$ of sugar. Fungi and other microorganisms were not seen in the cerebrospinal fluid and no organism grew on the cultures. The skull X-ray and carotid angiogram revealed no significant abnormalities. The electroencephalogram showed diffuse theta activity with a small amount of delta activity; no localized abnormality was demonstrated.

Clinical course: $\mathrm{He}$ was treated with steroids and antibiotics. The body temperature returned to normal two weeks later; consciousness improved gradually and he became alert in four weeks. A diffuse muscle atrophy was noticed in both legs on the seventh day of admission. The serum CPK was re-examined and was again normal. The electromyography with concentric needle electrodes showed in the right quadriceps femoris muscle, a decreased number of neuromuscular unit (NMU) potentials. Some of the individual NMU potentials had low amplitude and short duration. Positive sharp waves were recorded in the right tibialis anterior muscle, in which no potentials were obtained when voluntary contraction was attempted. The motor nerve conduction velocity of the
Table 1. Histochemical characteristics of basophilic material and eosinophilic mass.

$\begin{gathered}\text { Methods of } \\ \text { staining }\end{gathered}$
Haematoxylin and eosin
$\quad \begin{gathered}\text { haematoxylin } \\ \text { eosin }\end{gathered}$
Modified trichrome
P A S
DPNH-tetrazolium
reductase
Succinic dehydrogenase
Aldolase
Acid phosphatase
Myosin ATPase
Phosphorylase
Sudan black B
+++ strong staining
++ moderate staining

Staining intensity $\underset{\substack{\text { Basophilic } \\ \text { material }}}{\substack{\text { Eosinophilic } \\ \text { mass }}}$

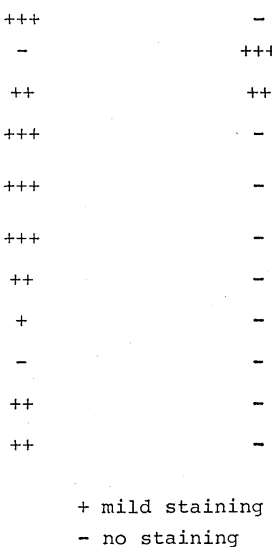

right tibial nerve was $50 \mathrm{~m} / \mathrm{sec}$ (normal).

Steroid therapy was continued and combined with physiotherapy. The motor and sensory disturbances recovered steadily. $\mathrm{He}$ was discharged six months later with only a slight difficulty in micturition. Otherwise there are no sequelae. Throughout the course of the illness, neither spasticity of the lower limbs nor extensor plantar responses were observed. The serum antibody titres against following viruses were measured on admission and three weeks later; influenza $\mathrm{A}$ and $\mathrm{B}$, parainfluenza, measles, mumps, rubella, respiratory syncytial, coxsackie $A$ and $B$, echo, Japanese B encephalitis, adeno, herpes simplex, herpes zoster and cytomegaloviruses. None of them showed signifi cant elevation.

\section{MATERIAL AND METHODS}

On the eighteenth day of admission, a muscle biopsy was taken under local anaesthesia from the vastus lateralis of the left quadriceps femoris muscle. Part of the biopsy was frozen and sections $8 \mu \mathrm{m}$ thick were cut on the cryostat. They were stained with haematoxylin and eosin, modified trichrome, periodic acid Schiff (PAS), DPNH (NADH)-tetrazolium reductase, suc- 
cinic dehydrogenase, aldolase, acid phosphatase, myosin ATPase ( $\mathrm{PH}$ 9.4), phosphorylase and Sudan black B. Another strip of muscle was removed in an isometric clamp in order to avoid contraction artifact, and fixed in 5\% glutaraldehyde and post-fixed in $2 \%$ osmium tetroxide. Thick sections of this material were stained with toluidine blue and examined with light microscope. Thin sections were stained with uranyl acetate and lead citrate, and examined by electron microscopy.

\section{OBSERVATION}

Light microscopy: Atrophied angular muscle fibres (Fig. 1,3), some of which forming small groups, were observed. In addition, fibres of round contour showing wide variation in diameter were conspicuous in some regions. Some fibres contained vacuoles (Fig. 2) and basophilic material or eosinophilic mass (Fig. 3). The interstitial tissue was widened. Some of the nerve fasciculi

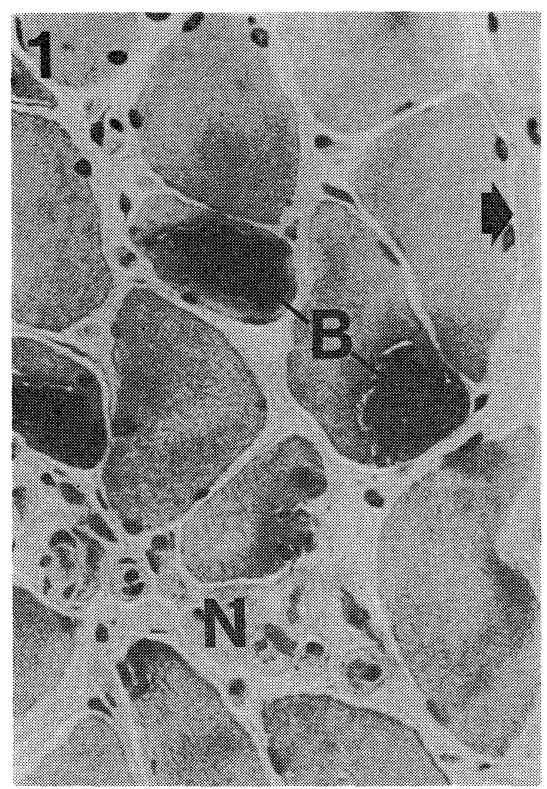

Fig. 1. While some of the atrophied muscle fibres had round contour, others were angular in shape (arrow). The muscle fibres with the basophilic material (B) were often nearby the intramuscula nerve fibres $(\mathrm{N})$. Proliferation of the Schwnan cells was noticed in the nerve fibres. (Modified trichrome stain. $\times 200$ )

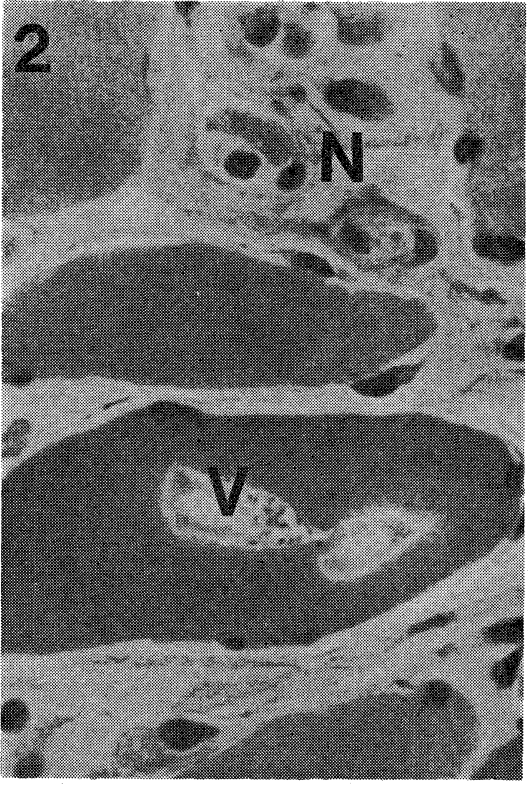

Fig. 2. Vacuoles (V) commonly occurred in the muscle fibres. (Modified trichrome stain. $\times 400)$

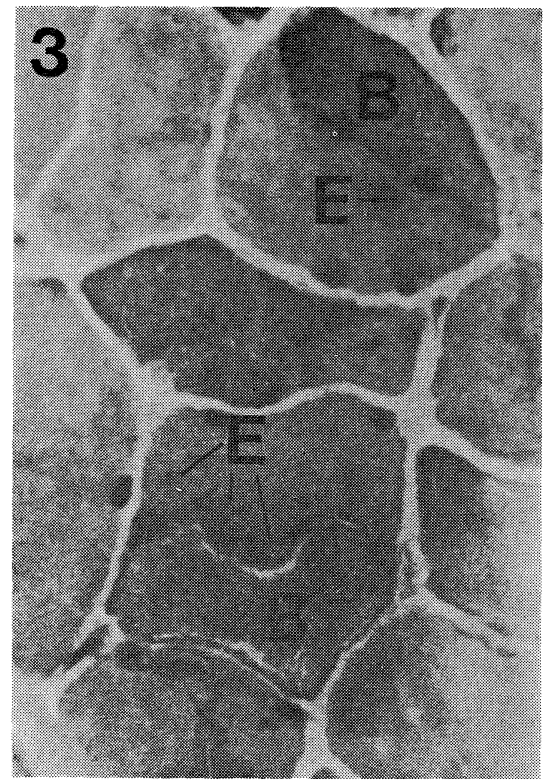

Fig. 3. The basophilic material (B) usually had granular appearance. The eosinophilic mass (E) was often present in the same muscle fibre. (Haematoxylin and eosin. $\times 400$ )

present in the muscle showed proliferation of the Schwann cell nuclei (Fig. 1). The blood vessels were normal and showed no 
perivascular cellular infiltration. A few degenerating muscle fibres contained phagocytes.

Many of the muscle fibres with vacuoles, basopilic material or eosinophilic mass contained more than one of these structures. Such muscle fibres tended to be adjacent to each other and frequently were near the intramuscular nerve fasciculi. The basopilic material, which was usually located at the periphery of the muscle fibres, had a granular appearance in many occasions. It stained reddish blue with the modified trichrome stain, showed high activity of DPNH-tetrazolium reductase and was positive with PAS and Sudan black B stain (Fig. 3 6). It had a moderate phosphorylase activity and no myosin ATPase activity (Fig. 7, 8). The eosinophilic mass was homogeneous and characterized by low enzymatic activities (Fig. 3,4). There was no evidence of fibre type atrophy or fibre type grouping. The results of the histochemical investigations are summarized in the table.

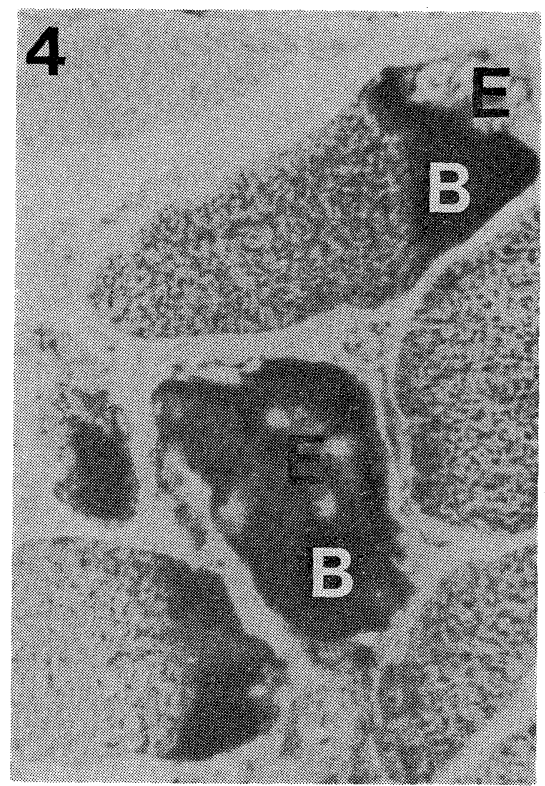

Fig. 4. The basophilic material (B) showed high activity of DPNH-tetrazolium reductase, while the eosinophilic mass (E) had low activity. $($ DPNH-tetrazolium reductase. $\times 400$ )

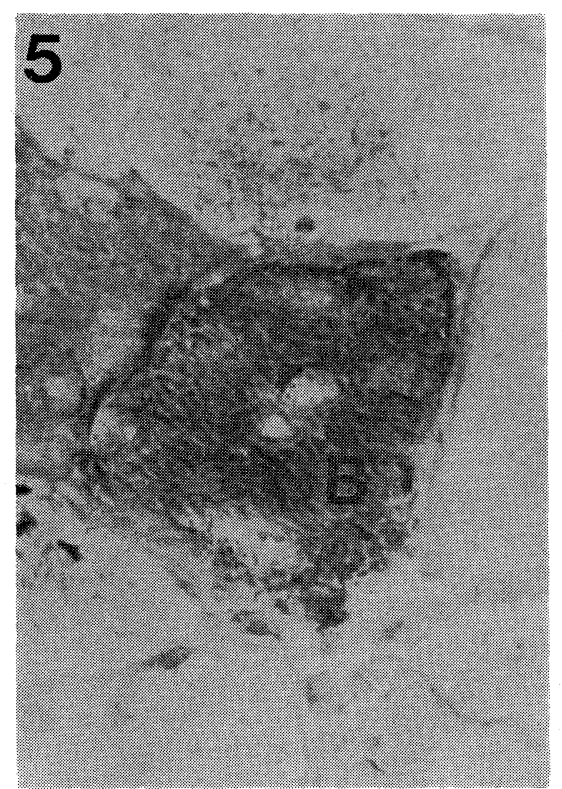

Fig. 5. The periodic acid Schiff stain showed positive substance in the basophilic material (B). (Periodic acid Schiff stain. $\times 400$ )

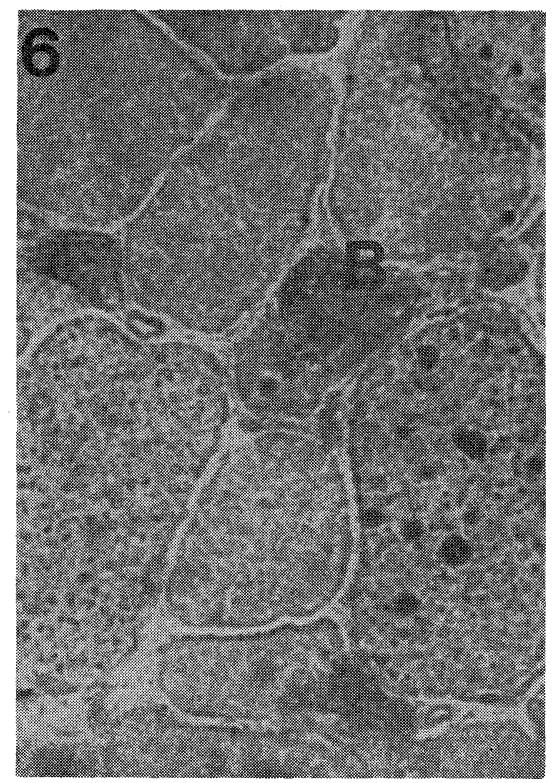

Fig. 6. The Sudan black B stain revealed a moderate reaction in the basophilic material (B). (Sudan black B stain. $\times 400$ )

Electron microscopy: (Fig. 9) Disarray and loss of myofibrils were marked. The autophagic vacuoles were frequent and some of them contained myelin figures. 


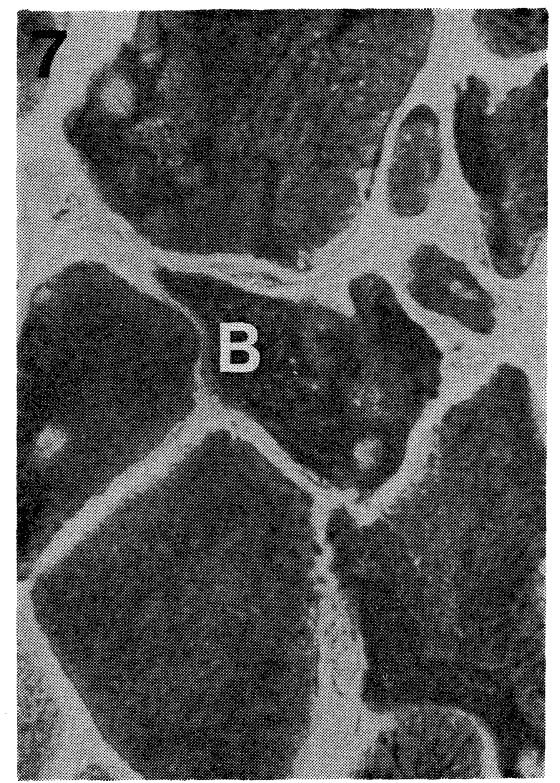

Fig. 7. A moderate activity of phosphorylase was observed in the basophilic material (B). (Phosphorylase. $\times 400$ )

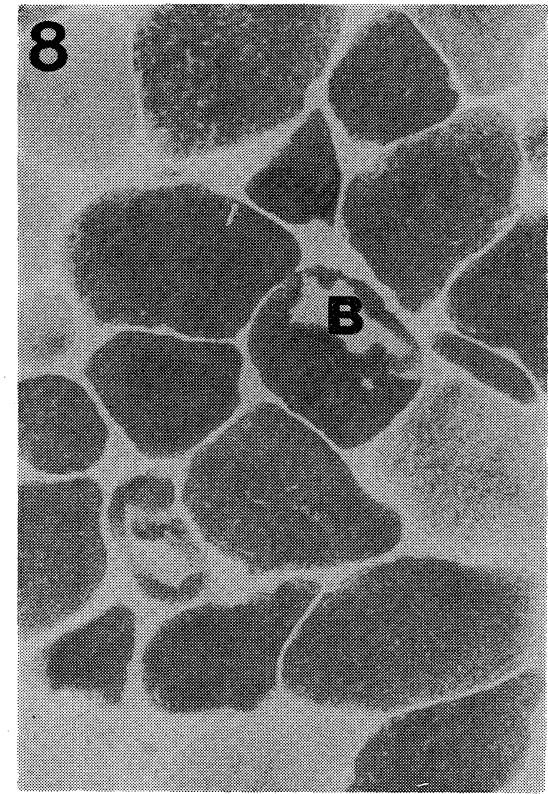

Fig. 8. Myosin ATPase activity was absent in the basophilic material (B). (Myosin ATPase. $\times 400$ )

The glycogen granules and mitochondria were abundant and crystalline inclusions were present in a few of the mitochondria.

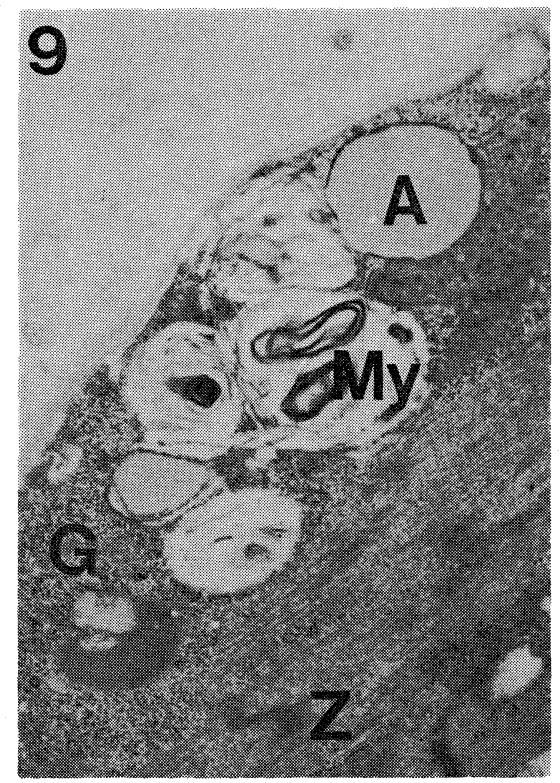

Fig. 9. The electron micrograph showed streaming of the $\mathrm{Z}$ lines $(\mathrm{Z})$ and accumulation of the glycogen granules $(G)$. The autophagic vacuoles (A) were frequently seen and some of them contained myelin figure (My). (Uranyl acetate and lead citrate. $\times 17,000$ )

Honeycomb structures were also seen. These findings were more conspicuous in the subsarcolemmal region in comparison to the central part of the muscle fibres.

\section{DISCUSSION}

In the present case, the rapid development of neurological manifestations due to wide spread lesions were preceded by a nonspecific upper respiratory tract infection. These features are in keeping with a diagnosis of ADEM. Although the differentiation of ADEM from acute multiple sclerosis is not always distinct, the presence of fever and flaccid paralysis are thought to be more in favour of ADEM than acute multiple sclerosis ${ }^{2}$. Poliomyelitis is an unlikely diagnosis because of the profound sensory impairment. No evidence of infection of any particular virus was obtained on laboratory examination. The pathogenesis of ADEM is not certain, but the role of a hypersensitivity reaction has

Jap J Med Vol 19, No 3 (July 1980) 
been suggested by Poser $^{4)}$ and many other authors.

Among 34 cases of ADEM reported by Miller and Evans ${ }^{1)}$, one case (case 13) was associated with polyradiculitis and showed wasting and tenderness of the muscle. McAlpine $^{2)}$ mentioned one probable case of muscle atrophy in his series of ADEM. van Bogaert ${ }^{3)}$ saw, in one (case 2) of the autopsied cases of ADEM, hyalinization of a few muscle fibres, and leukocytes among the muscle fibres. More recently, cases with muscular symptoms such as myalgia and weakness, in association with encephalomyelitic disturbance of generally benign nature, have been reported under various names, e.g. Icelandic disease, benign myalgic encephalomyelitis and Royal Free disease $^{5)}$.

The small angular muscle fibres seen in the biopsied muscle indicate neuropathic change, while the round atrophied fibres are suggestive of myopathic change. Vacuolar degeneration is encountered in various myopathies including polymyositis, steroid myopathy, trauma and others ${ }^{6)}$. Since the patient recovered despite the continuation of steroid therapy, steroid induced myopathy therefore seems unlikely. Trauma is also unlikely to be responsible because of the diffuse symmetrical distribution of the muscle atrophy. As to the basophilic material seen in haematoxylin and eosin stain, reddish colour seen in the modified trichrome stain and high activity of oxidative enzymes such as DPNHtetrazolium reductase are suggestive of the accumulation of mitochondria ${ }^{7}$. Positive PAS and the Sudan black B reaction imply the presence of carbohydrate and lipid respectively. These findings correspond presumably to the abundant mitochondria, glycogen granules and myelin figures seen in the electron micrographs. In the early stage of muscle regeneration, the sarcoplasm is strongly basophilic and shows low myosin ATPase and high oxidative enzymatic activity; the phosphorylase activity is irregular and often absent according to
Reznik $^{8)}$. These features have considerable similarity to the basophilic material in this case. On the other hand, the homogeneous structure and low enzymatic activities of the eosinophilic mass are consistent with hyaline degeneration.

The cause of the muscle atrophy in this case is uncertain. On account of the suppressed tendon reflexes, which persisted in the lower limbs after the acute stage of the disease in the present case, the nerve roots and or the peripheral nerves are likely to have been involved in addition to the central nervous system. The normal motor conduction velocity of the nerve does not rule out a possibility of a limited degree of damage to the peripheral nerve. Thus the mild neuropathic changes in the muscle in the present case can be attributed to the impairment of the nerve roots and the peripheral nerves. Howeves, degenerative changes of the muscle fibres such as vacuolar and hyaline degeneration are unusual in the early stage of denervation in human muscles ${ }^{9)}$. Immobilization or diuse of the muscle in man usually results in simple atrophy, i.e. reduction of the muscle fibre diameter. Only a limited degree of degenerative changes have been reported in a small number of examples of disuse atrophy ${ }^{10)}$. In some animal models of disuse atrophy and denervation atrophy, however, various degenerative changes have been described ${ }^{11)}$. W.K. Engel and coworkers ${ }^{12)}$, in tenotomized muscle of the cats, saw sarcoplasmic mass which was stained red with the modified trichrome stain and had high activity of oxidative enzymes but no activity of myosin ATPase. These histochemical features have some similarity to the basophilic material in the present case. It is, therefore, probably not impossible to attribute the vacuolar and hyaline degeneration of the muscle in the present case to immobilization and denervation.

Another possible cause of the muscle changes is polymyositis. Degeneration and regeneration of the muscle fibres are often 
prominent in this disorder ${ }^{13)}$. The absence of any cellular infiltration in the interstitial tissue leaves this possibility unconfirmed. Nevertheless, the extent of the cellular infiltration in polymyositis is so variable that infiltrates are even absent in some clinically typical cases ${ }^{14}$. Mastaglia and Kakulas $^{15)}$ described vacuolar degeneration, hyaline degeneration and regeneration of the muscle fibres in a case of polymyositis. The histochemical features of the regenerating fibres in their case are similar to those of the basophilic material of the present case except for the phosphorylase and acid phosphatase activities; the activities of the latter two enzymes were absent in the regenerating fibre described by Mastaglia and Kakulas while they are present in the basophilic material of the present case. Carpenter, Karpati and Wolfe ${ }^{16)}$ reported a case of chronic myopathy which showed virus-like filamentous inclusions and phospholipid accumulation in the skeletal muscle. They also saw vacuolar degeneration, basophilic material and eosinophilic mass in the muscle fibres, but cellular infiltration was not seen in the interstitial tissue. Their patient had a prolonged history of ten years' duration in contrast with the short history of the present case. Since there has been some evidence to suggest that delayed hypersensitivity is responsible for polymyositis ${ }^{17)}$, it may be possible to suspect that both ADEM and some of the changes of the muscle of the present case have resulted from a delayed hypersensitivity reaction. Viral infection has been also discussed as a possible cause of polymyositis based on the finding of virus-like inclusions in some $\operatorname{cases}^{18)}$. However, we could not obtain enough evidence indicative of viral infection of the muscle in the present case.

\section{REFERENCES}

1) Miller H, Evans MJ: Prognosis in acute disseminated encephalomyelitis with a note on neuromyelitis optica. Quart J Med 22: $347,1953$.
2) McAlpine D: Acute disseminated encephalomyelitis. Its sequelae and its relationship to disseminated sclerosis. Lancet 1: 846, 1931.

3) van Bogaert L: Post-infectious encephalomyelitis and multiple sclerosis. The significance of perivenous encephalomyelitis. J Neuropath exp Neurol 9: 219, 1950.

4) Poser CM: Disseminated vaculomyelinopathy. A review of the clinical and pathologic reactions of the nervous system in hyperergic diseases. Acta neurol Scand 45 (suppl. 37) : 1, 1969.

5) Ramsay AM, Dowsett EG, Dadswell JV, et al: Icelandic disease (benign myalgic encephalomyelitis or Royal Free disease). Brit med J 1 (6072) : 1350, 1977.

6) Engel AG : Vacuolar myopathies. Multiple etiologies and sequential structural studies, chap 16. The Striated Muscle (Pearson CM, Mostofi FK, eds), Williams \& Wilkins, Baltimore, 1973.

7) Dubowitz V, Brooke MH: Muscle Biopsy. A Modern Approach, Saunders, London, 1973.

8) Reznik M: Current concept of skeletal muscle regeneration, chap 10 . The Striated Muscle (Pearson CM, Mostofi FK, eds), Williams \& Wilkins, Baltimore, 1973.

9) Gutmann E, Zelená J : Morphological changes in the denervated muscle. The Denervated Muscle (Gutmann E, ed) Publishing House of the Czechoslovak Academy of Science, Prague, p 57, 1962.

10) Sunderland S, Lavarack JD: Changes in human muscle after permanent tenotomy. J Neurol Neurosurg Psychiat 22: 167, 1959.

11) Gutmann E: Denervation and disuse atrophy in cross-striated muscle. Rev Canad Biol $21: 353,1962$.

12) Engel WK, Brooke MH, Nelson PG: Histochemical studies of denervation or tenotomized cat muscle; illustrating difficulties in relating experimental animal conditions to human neuromuscular diseases. Ann N Y Acad Sci 138 : 160, 1966.

13) Matsubara S: Ultrastructural changes in polymyositis and related disorders. $\mathrm{Ph}$. D. Thesis, London University, 1978.

14) Pearson CM, Currie S: Polymyositis and related disorders, chap 16. Disorders of Voluntary Muscle, 3 rd ed (Walton JN ed), Churchill Livingstone, Edinburgh, 1974. 
15) Mastaglia FL, Kakulas BA : A histological study of skeletal muscle regeneration in polymyositis. J neurol Sci 10: 471, 1970.

16) Carpenter S, Karpati G, Wolfe L: Virus. like filaments and phospholipid accumulation in skeletal muscle. Study of histochemically distinct chronic myopathy. Neurology (Minneap.) $20: 889,1970$.
17) Currie S, Saunders $M$, Knowels $M$, et al: Immunological aspects of polymyositis : The in vitro activity of lymphocytes on incubation with muscle antigen and with muscle cultures. Quart J Med 40:63, 1971.

18) Ghou SM: Myxovirus-like structures in a case of human chronic polymyositis. Science $158: 1453,1967$. 